\title{
Does thermodynamics improve processing when chemical reaction is integrated with physical separation in binary ideal mixtures?
}

\section{INTRODUCTION}

Chemical engineering practice has been traditionally faced with the demand for nonexisting compounds possessing predetermined purity specifications; while such compounds can, in principle, be produced via chemical reaction from suitable feedstocks, the upper limits on conversion arising from chemical equilibrium considerations are often overcome through implementation of downstream physical separation The classical approach to this issue is to consider reaction and separation as independent but sequential unit operations, in what may be viewed as resulting from a macroscopic rationale. However, since the phenomena in question (i.e. mixing, separation and transformation) occur on the molecular level, design of processes that do not allow bulk concentrations of products to build up seems promising, and the concept of integrating reaction and separation (in what may be viewed as resulting from a microscopic rationale) has consequently been under scrutiny by an increasing number of researchers in recent years. The topic of extractive reaction, or reaction coupled with in situ separation, has been claimed to provide kinetic enhancement and thermodynamic enhancement especially in the case of biochemical processes (e.g. Laane et al., 1987; Tramper et al., 1992). Most systems studied to date consist of sets of two essentially immiscible liquid phases where one plays the role of reactant source and the other of product sink, and there seems to be a trend towards reducing the intrinsic substrate-control upon the reaction rate via solvent-free systems which keep substrates as concentrated as possible.

The claims that integration of reaction and separation brings about thermodynamic improvement of the process by shifting the reaction equilibrium are essentially qualitative in nature and consider the point of view only of the reaction rather than the point of view of the overall process (i.e reaction and separation). If a quantitative thermodynamic description of the overall process via a suitable function of state is considered, then first principles make it obvious that, for given pressure, temperature, and composition of the inlet and outlet streams, no difference exists between sequential and simultaneous reaction and separation, irrespective of how the detail of the process is arranged. This Communication intends to shed extra light over these matters by proving that the lack of thermodynamic improvement associated with integrated reaction and separation (as measured by the Gibbs' free energy change of the overall process) results from exact cancellation of the reaction contribution by the corresponding separation contribution. Since a general liquid mix ture containing several components and prone to nonideal behaviour is rather difficult to work out analytically, the simpler model system of a binary ideal reactant/product mix- ture was selected for this analysis. In order to further facilitate the analysis, pressure and temperature were assumed to remain steady and uniform (which justifies use of Gibbs' free energy as a quantitative measure), so the rationale is drawn solely upon compositional changes.

\section{MATHEMATICAL ANALYSIS}

When working in the thermodynamic domain, all systems are in states of mechanical, thermal, and chemical equilibrium, or evolve through states which are displaced only infinitesimally from them (i.e. reversible processes). In such domain time is not a relevant variable because momentum. heat and mass transfer phenomenological coefficients (i.e. conductances) are not thermodynamic variables. If an isothermal and isobaric process is considered, then the price one has to pay to bring about said process is preferably measured by Gibbs' free energy change, a quantity that is commonly used as criterion of equilibrium, viz. $(\mathrm{d} G)_{P, T}=0$, and also as criterion for spontaneous events, viz. $(\Delta G)_{P, T}<0$.

Consider first the classical unit operation approach using a series of two apparatus, where the first is a reactor and the second a separator, as depicted in Fig. 1(a). The standard molar Gibbs' free energy associated with a chemical reaction that converts one mole of pure reactant, $R$, into one mole of pure product, $\mathrm{P}$, according to the chemical equation $\mathrm{R} \rightarrow \mathrm{P}$ at a given absolute temperature $T$ and at the reference pressure $P, \Delta g_{r}^{\theta}\{T\}$, up to an equilibrium conversion level $X_{\mathrm{eq}}$ in an ideal system is given by (Smith and van Ness, 1987)

$$
\frac{\Delta g_{r}^{\theta}\{T\}}{R T}=\ln \left\{\frac{1-X_{\mathrm{eq}}}{X_{\mathrm{eq}}}\right\}
$$

where $R$ is the ideal gas constant and $X_{\text {eq }} \equiv\left(n_{\mathrm{R}, q}-\right.$ $n_{\mathrm{R}, \text { eq}}$ ) $/ n_{\mathrm{R}, \mathrm{O}}$ (where $n_{\mathrm{R}}$ denotes the number of moles of reactant $R$, and subcripts eq and 0 denote equilibrium and inlet conditions, respectively). Recall the fundamental property relation for single-phase binary systems of $\mathrm{R}$ and $\mathrm{P}$ (Smith and van Ness, 1987), viz.

$$
\begin{aligned}
& \Delta G_{r}\left\{n_{0}, X_{\text {eq }}, T\right\} \\
& \quad=n_{0} \int_{0}^{X_{\mathrm{eu}}}\left(\mu_{\mathbf{P}}\{\Xi, T\}-\mu_{\mathrm{R}}\{\Xi, T\}\right) \mathrm{d} \Xi
\end{aligned}
$$

where $\Delta G_{r}\left\{n_{0}, X_{\text {eq }}, T\right\}$ denotes the total Gibbs' free energy associated with transformation by chemical reaction of $n_{0}$ initial moles of pure $\mathrm{R}$ into a mixture of $n_{0}\left(1-X_{\mathrm{eq}}\right)$ moles of $\mathrm{R}$ and $n_{0} X_{\mathrm{eq}}$ moles of $\mathrm{P}$, with $X_{\mathrm{eq}}$ denoting the equilibrium conversion calculated from eq. (1), and where $\mu_{\mathrm{R}}\{\Xi, T\}$ and $\mu_{\mathbf{P}}\{\Xi, T\}$ denote the chemical potential of reactant and 
product, respectively, at the molar fractions associated with conversion $\Xi$ and at absolute temperature $T$. The chemical potential of a compound in an ideal mixture may in turn be calculated by

$$
\mu_{i}\left\{x_{i}, T\right\}=g_{i}^{\theta}\{T\}+R T \ln x_{i}
$$

where $g_{i}^{\theta}\{T\}$ denotes Gibbs' free energy of species $i$ as a pure compound at temperature $T$ and $x_{i}$ denotes the molar fraction of the same species in the mixture. Combination of eqs (2) and (3) and integration yields (Gradshteyn and Ryzhik, 1980)

$$
\begin{array}{r}
\frac{\Delta G_{r}\left\{n_{0}, X_{\mathrm{eq}}, T\right\}}{R T}=n_{0}\left(\frac{\Delta g_{r}^{\theta}\{T\}}{R T} X_{\mathrm{eq}}+X_{\mathrm{eq}} \ln \left\{X_{\mathrm{eq}}\right\}\right. \\
\left.+\left(1-X_{\mathrm{eq}}\right) \ln \left\{1-X_{\mathrm{eq}}\right\}\right) .
\end{array}
$$

Further combination with eq. (1) allows eq. (4) to be simplified to

$$
\frac{\Delta G_{r}\left\{n_{\mathrm{o}}, X_{\mathrm{eq}}, T\right\}}{R T}=n_{0} \ln \left\{1-X_{\mathrm{eq}}\right\} \text {. }
$$

The standard molar Gibbs' free energy associated with physical separation of an ideal binary mixture into its pure components at the aforementioned (reference) temperature, $\Delta g_{s}^{\theta}\left\{x_{\mathrm{R}}, T\right\}$, is given by (Smith and van Ness, 1987)

$$
-\frac{\Delta g_{s}^{\theta}\left\{x_{R}, T\right\}}{R T}=x_{R} \ln \left\{x_{R}\right\}+\left(1-x_{R}\right) \ln \left\{1-x_{R}\right\}
$$

Hence, the total Gibbs' free energy associated with physical separation of a mixture containing $n_{0}$ total moles of reactant and product (assumed to be at chemical equilibrium) at the outlet of the reactor into their pure components, $\Delta G_{s}\left\{n_{0}, X_{c q}, T\right\}$, should read

$$
\begin{aligned}
\frac{\Delta G_{s}\left\{n_{0}, X_{\text {eq }}, T\right\}}{R T}= & -n_{0}\left[\left(1-X_{\text {eq }}\right) \ln \left\{1-X_{\text {eq }}\right\}\right. \\
& \left.+X_{\text {eq }} \ln \left\{X_{\text {eq }}\right\}\right] .
\end{aligned}
$$

From combination of eqs (4) and (7), the overall Gibbs' free energy associated with the reaction/separation process, $\Delta G_{r+s}\left\{n_{0}, X_{\mathrm{eq}}, T\right\}$, will read

$$
\begin{aligned}
\frac{\Delta G_{r+s}\left\{n_{0}, X_{\mathrm{eq}}, T\right\}}{R T} & \equiv \frac{\Delta G_{r}\left\{X_{\mathrm{eq}}, T\right\}}{R T}+\frac{\Delta G_{s}\left\{X_{\mathrm{eq}}, T\right\}}{R T} \\
& =n_{0} X_{\mathrm{eq}} \ln \left\{\frac{1-X_{\mathrm{eq}}}{X_{\mathrm{eq}}}\right\} .
\end{aligned}
$$

Consider next an intensification of the classical unit operation approach given by a cascade of $N$ pairs of apparatus, each consisting of a reactor and a separator placed in series, as depicted in Fig. 1(b). Chemical equilibrium conditions are assumed to prevail at the $i$ th reactor, so the inlet pure reactant, $n_{\mathrm{o}}\left(1-X_{\mathrm{eq}}\right)^{i-1}$, is assumed to be transformed into a mixture containing $n_{0}\left(1-X_{\mathrm{eq}}\right)^{i}$ of $\mathrm{R}$ and $n_{0}\left(1-X_{\mathrm{eq}}\right)^{i-1} X_{\mathrm{eq}}$ of P. Applying eq. (5) sequentially to this situation, the total Gibbs' free energy associated with chemical transformation within the $i$ th reactor, $\Delta G_{r, i}\left\{n_{0}, X_{\text {eq }}, T\right\}$, should read

$$
\begin{array}{r}
\frac{\Delta G_{r, i}\left\{n_{0}, X_{\mathrm{eq}}, T\right\}}{R T}=n_{0}\left(1-X_{\mathrm{eq}}\right)^{i-1} \ln \left\{1-X_{\mathrm{eq}}\right\}, \\
i=1,2, \ldots, N
\end{array}
$$

where $X_{\mathrm{eq}} \equiv\left(n_{\mathrm{R}, i-1}-n_{\mathrm{R}, i, \mathrm{eq}}\right) / n_{\mathrm{R}, i-1}$ (where subscripts $i$ and $i-1$ denote the outlet from, and the inlet to, the $i$ th reactor, respectively). Recalling the expression for the sum of the first $N$ terms of a geometric series (Gradshteyn and Ryzhik, 1980), the overall Gibbs' free energy associated with chemical transformation, $\Delta G_{R}\left\{N, n_{0}, X_{\mathrm{eq}}, T\right\}$, then becomes

$$
\begin{aligned}
\frac{\Delta G_{R}\left\{N, n_{0}, X_{\mathrm{eq}}, T\right\}}{R T} \equiv & \sum_{i=1}^{N} \frac{\Delta G_{r, i}\left\{n_{0}, X_{\mathrm{eq}}, T\right\}}{R T} \\
= & n_{0}\left(1-\left(1-X_{\mathrm{eq}}\right)^{N}\right) \\
& \times \frac{\ln \left\{1-X_{\mathrm{eq}}\right\}}{X_{\mathrm{eq}}} .
\end{aligned}
$$

Now applying eq. (7) sequentially to this cascading situation, the total Gibbs' free energy associated with physical separation of the reactant and product as pure components from the outlet of the $i$ th reactor, $\Delta G_{s, i}\left\{n_{0}, X_{\text {eq }}, T\right\}$, should read

$$
\begin{aligned}
& \frac{\Delta G_{s, i}\left\{n_{0}, X_{\mathrm{eq}}, T\right\}}{R T} \\
& =-n_{0}\left(1-X_{\mathrm{eq}}\right)^{i-1}\left[\left(1-X_{\mathrm{eq}}\right) \ln \left\{1-X_{\mathrm{eq}}\right\}\right. \\
& \left.\quad+X_{\mathrm{eq}} \ln \left\{X_{\mathrm{eq}}\right\}\right] .
\end{aligned}
$$

Recalling again the expression for the sum of the first $N$ terms of a geometric series, the overall Gibbs' free energy associated with physical separation of the mixture of reactant and product (assumed to be at chemical equilibrium) at the outlet of the $i$ th reactor into their pure components,

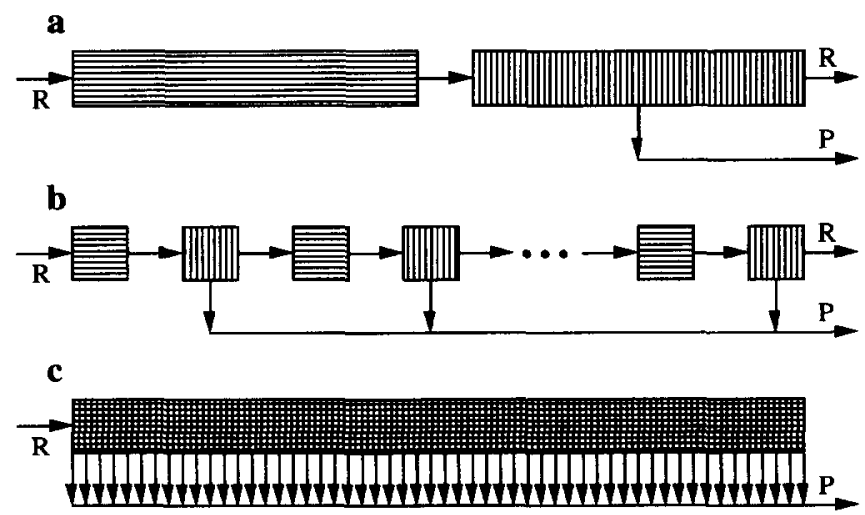

Fig. 1. Combination of reactor (目) and separator (牦) units for production of product $\mathbf{P}$ from reactant $R$ following (a) a unit operation approach, (b) a cascaded operation approach, and (c) an integrated operation approach. 
$\Delta G_{S}\left\{N, n_{0}, X_{\text {eq }}, T\right\}$, then becomes

$$
\begin{aligned}
& \frac{\Delta G_{S}\left\{N, n_{0}, X_{\mathrm{eq}}, T\right\}}{R T} \\
& \equiv \sum_{i=1}^{N} \frac{\Delta G_{\mathrm{s}, i}\left\{n_{0}, X_{\mathrm{eq}}, T\right\}}{R T}=-n_{0}\left[1-\left(1-X_{\mathrm{eq}}\right)^{N}\right] \\
& \quad \times \frac{X_{\mathrm{eq}} \ln \left\{X_{\mathrm{eq}}\right\}+\left(1-X_{\mathrm{eq}}\right) \ln \left\{1-X_{\mathrm{eq}}\right\}}{X_{\mathrm{eq}}} .
\end{aligned}
$$

The overall Gibbs' free energy associated with the reaction/separation brought about in the $i$ th reactor/separator pair, $\Delta G_{r+s, i}\left\{n_{0}, X_{\mathrm{eq}}, T\right\}$, will, in view of eqs (9) and (11), read

$$
\begin{aligned}
& \frac{\Delta G_{r+s, i}\left\{n_{0}, X_{\mathrm{eq}}, T\right\}}{R T} \\
& \equiv \frac{\Delta G_{r, i}\left\{n_{0}, X_{\mathrm{eq}}, T\right\}}{R T}+\frac{\Delta G_{s, i}\left\{n_{0}, X_{\mathrm{eq}}, T\right\}}{R T} \\
& =n_{0} X_{\mathrm{eq}}\left(1-X_{\mathrm{eq}}\right)^{i-1} \ln \left\{\frac{1-X_{\mathrm{eq}}}{X_{\mathrm{eq}}}\right\} .
\end{aligned}
$$

Applying eq. (13) sequentially, the overall Gibbs' free energy associated with chemical reaction and physical separation of reactant and product as pure components in the whole cascade, $\Delta G_{R+S}\left\{N, n_{0}, X_{\mathrm{eq}}, T\right\}$, should read

$$
\begin{aligned}
\frac{\Delta G_{R+s}\left\{N, n_{0}, X_{\mathrm{eq}}, T\right\}}{R T} \equiv & \sum_{i=1}^{N} \frac{\Delta G_{r+s, i}\left\{n_{0}, X_{\mathrm{eq}}, T\right\}}{R T} \\
= & n_{0}\left[1-\left(1-X_{\mathrm{eq}}\right)^{N}\right] \\
& \times \ln \left\{\frac{1-X_{\mathrm{eq}}}{X_{\mathrm{eq}}}\right\} .
\end{aligned}
$$

On the other hand, the amount of product $P$ recovered in pure form via the physical separation process in the $i$ th separator, $n_{\mathrm{P}, i}\left\{n_{0}, X_{\mathrm{eq}}\right\}$, is given by

$$
n_{\mathrm{P}, i}\left\{n_{0}, X_{\mathrm{eq}}\right\}=n_{0} X_{\mathrm{eq}}\left(1-X_{\mathrm{eq}}\right)^{i-1}
$$

and so the total amount recovered in the $N$ separator unit, $n_{\mathrm{P}}\left\{N, n_{0}, X_{\mathrm{cq}}\right\}$, is given by

$$
\begin{aligned}
n_{\mathrm{P}}\left\{N, n_{0}, X_{\mathrm{eq}}\right\} & \equiv \sum_{i=1}^{N} n_{\mathrm{p}, i}\left\{n_{0}, X_{\mathrm{eq}}\right\} \\
& =n_{0}\left[1-\left(1-X_{\mathrm{eq}}\right)^{N}\right] .
\end{aligned}
$$

Consider finally the case of complete integration of reaction and separation. This limiting situation corresponds to an infinite degree of reactor/separator cascading; therefore, the overall Gibbs' free energy associated with chemical transformation, $\Delta \widehat{G_{R}}\left\{n_{0}, X_{\text {eq }}, T\right\}$, can be obtained from eq. (10) as

$$
\begin{aligned}
\frac{\Delta \widehat{G_{R}}\left\{n_{0}, X_{\mathrm{eq}}, T\right\}}{R T} & \equiv \lim _{N \rightarrow \infty} \frac{\Delta G_{R}\left\{N, n_{0}, X_{\mathrm{eq}}, T\right\}}{R T} \\
& =n_{0} \frac{\ln \left\{1-X_{\mathrm{eq}}\right\}}{X_{\mathrm{eq}}}
\end{aligned}
$$

By the same token, the overall Gibbs' free energy associated with physical separation of the mixture of reactant and product (assumed to be at a state of local chemical equilibrium) into their pure components, $\Delta \widehat{G_{s}}\left\{n_{0}, X_{e q}, T\right\}$, can be obtained from eq. (12) as

$$
\begin{aligned}
& \frac{\Delta \widehat{G_{S}}\left\{n_{0}, X_{\mathrm{eq}}, T\right\}}{R T} \equiv \lim _{N \rightarrow x} \frac{\Delta G_{S}\left\{N, n_{0}, X_{\mathrm{eq}}, T\right\}}{R T} \\
& =-n_{0} \frac{X_{\mathrm{eq}} \ln \left\{X_{\mathrm{eq}}\right\}+\left(1-X_{\mathrm{eq}}\right) \ln \left\{1-X_{\mathrm{eq}}\right\}}{X_{\mathrm{eq}}} .
\end{aligned}
$$

Following the same reasoning once more, the overall Gibbs' free energy associated with chemical reaction and physical separation of reactant and product as pure components, $\Delta \overline{G_{R+s}}\left\{n_{0}, X_{\text {eq }}, T\right\}$, can be obtained from eq. (14) as

$$
\begin{aligned}
\frac{\Delta \widehat{G_{R+S}}\left\{n_{0}, X_{\mathrm{eq}}, T\right\}}{R T} & \equiv \lim _{N \rightarrow \infty} \frac{\Delta G_{R+s}\left\{N, n_{0}, X_{\mathrm{eq}}, T\right\}}{R T} \\
& =n_{0} \ln \left\{\frac{1-X_{\mathrm{eq}}}{X_{\mathrm{eq}}}\right\} .
\end{aligned}
$$

Finally, the total amount of pure product $\mathbf{P}$ recovered, $\widehat{n_{\mathrm{P}}}\left\{n_{0}, X_{\mathrm{cq}}\right\}$, can be obtained from eq. (16) as

$$
\widehat{n_{\mathbf{P}}}\left\{n_{0}, X_{\text {eq }}\right\} \equiv \lim _{N \rightarrow \infty} n_{\mathbf{P}}\left\{N, n_{0}, X_{\text {eq }}\right\}=n_{0} .
$$

Combination of eqs (1), (8), (14), (16), (19), and (20) finally yields

$$
\begin{aligned}
& \frac{\Delta G_{r+s}\left\{N, n_{0}, X_{\mathrm{cq}}, T\right\}}{R T} \\
& n_{0} X_{\mathrm{eq}} \\
& =\frac{\Delta G_{R+s}\left\{N, n_{0}, X_{\mathrm{eq}}, T\right\}}{R T} \\
& =\ln \left\{\frac{1-X_{\mathrm{eq}}\left\{N, n_{0}, X_{\mathrm{eq}}\right\}}{X_{\mathrm{eq}}}\right\}=\frac{\Delta g_{\mathrm{r}}^{\theta}\{T\}}{R T}
\end{aligned}
$$

which reinforces the idea that the variation of the molar overall Gibbs' free energy associated with a given chemical reaction carried out at given pressure and temperature is the same irrespective of whether the reaction and separation steps are taken sequentially or are (partially or fully) integrated.

\section{DISCUSSION AND CONCLUSIONS}

Although the thermodynamic analysis presented in this communication is in principle valid for any form or configuration of reactor/separator, it is particularly easy to provide a physicochemical rationale if the separation process is devised as a pressure-driven process. In fact, it may be considered that product $\mathbf{P}$ differs from reactant $\mathbf{R}$ in terms of shape or size to such a low degree that their mixture behaves ideally but to such a high degree that an ultra- or nanofiltration membrane providing essentially complete molecular exclusion of $P$ relative to $R$ can be suitably designed and constructed. In this situation, reaction would be effected up to equilibrium conversion in each reactor unit and recovery of $\mathbf{P}$ in pure form from the outlet of said reactor unit would be accomplished via the permeate in the separator unit located adjacent [see Fig. 1(b)] provided that a sufficiently high work input in the form of overpressure on the retentate side would be available and that a sufficiently high tangential flow rate would be possible so as to avoid significant fouling of the membrane. The integrated situation [see Fig. 1(c)] would thus correspond to performing the chemical reaction on the retentate side (or, at most, on both the retentate side and the inner hold-up volume) of said membrane; if the reaction required a catalyst, then it should be immobilized onto the inner surface of the membrane (or onto the inner surface of the membrane pores). Such a membrane device would possess the further advantage of exhibiting a high thermodynamic efficiency when compared with alternative separation devices based on indirect supply of work (e.g. thermal engines as in distillation).

The backbone of the rationale followed in the analysis presented above is that integration [as depicted in Fig. 1(c)] results from intensification of the unit operation approach [as depicted in Fig. 1(b)] up to its uppermost limit; therefore, the integrated system may be viewed mathematically as the limit of cascading reaction and separation when the number of reactor/separator pairs becomes infinite. Although in practice kinetic limitations are ubiquitously 
present to some extent, they are not considered in the thermodynamic domain and so prediction of the reactor and separator sizes cannot be made based solely on thermodynamic information. An actual situation that will approximately meet the assumptions of our analysis is that where the time scales associated with molecular transport in the reactor and in the separator are negligible when compared with the time scale associated with chemical reaction, and where the time scale associated with chemical reaction is in turn sufficiently small that the composition of the reacting system in the reactor can be obtained in all cases from plain application of Guldberg and Waage's law of mass action. In this situation the sizes of the reactor and separator able to bring about a given reaction/separation process are quite small, and so these units can be viewed as a differential reactor and a differential separator, respectively; therefore, cascading the reactor with the separator (i.e. performing reaction and separation sequentially, yet at distinct locations) with a large number of units would be equivalent to recovering in situ the desired product (i.e. performing reaction and separation si. multaneously, at adjacent locations). In such a case, distinction between local and bulk mole fractions vanishes, and so bulk mole fractions can still be used (as they were in the cascade approach) since in the limit they become coincident with local mole fractions. Furthermore, the aforementioned assumptions encompassing negligible time scales prevent concentration gradients from building up spontaneously anywhere in the reaction system; this means that chemical equilibrium conditions will be reached in the neighborhood of the catalyst when a differential amount of $\mathbf{R}$ is converted to a corresponding differential amount of $P$ (thus releasing, or absorbing, a differential amount of Gibbs' free energy) and mixing of the species in this neighborhood will immediately occur, whereas establishment of concentration gradients wil only be possible across the porous membrane due to application of external work.

It might seem somewhat paradoxical that integrated reaction/separation systems are not favored from a thermodynamic point of view relative to unit operation systems, irrespective of equilibrium conversion, when it is more or less obvious that if a reaction product can be continuously removed from the reaction system via, say, selective permeation, true equilibrium conditions are reached only locally and for an instant and so the reactant will be eventually and fully converted into product (unlike what happens in the unit operation approach where the reactant will only attain the equilibrium conversion to product). The reason for this apparent paradox is that our analysis encompasses the whole reaction/separation system and not the reaction system itself; although in the example thus described the overall extent of reaction is clearly improved (up to unity) by not allowing equilibrium conditions to be attained except in an infinitesimal portion of space and for an infinitesimal time period on the reaction side, the extra input of Gibbs' free energy in the form of work to bring about separation of the product from the unreacted reactant must be considered if full account for the global processing system is sought.

Although the conclusion that no thermodynamic enhancement can be claimed for integration, unlike what happens with respect to kinetic enhancement (Paiva et al., 1996), was based on assumption of an ideal binary mixture, extension to multicomponent ideal solutions is straightforward. On the other hand, assumption of nonideality will not affect this conclusion because the Gibbs' free energy of separation in the presence of activity coefficients different from unity is (as happened in our analysis) the negative of the term that must be added to $\Delta g_{r}^{\theta}\{T\}$ to obtain Gibbs' free energy of reaction; therefore, the result for the combined reaction and separation processes turns out to be, as expected, equal to the standard Gibbs' free energy of reaction, which is a constant for each system selected and hence does not depend on whether a unit operation approach or an integrated approach is considered.

\section{Acknowledgements}

The authors would like to acknowledge financial support provided by JNICT through programs CIENCIA (fellowship BD/2081/92-IF) and PRAXIS XXI (project 2/2.1/BIO/ 34/94, Extractive Biocatalysis).

\section{A. L. PAIVA}

F. X. MALCATA*

\author{
Escola Superior de Biotecnologia \\ Universidade Católica Portuguesa \\ Rua Dr. António Bernardino de Almeida \\ 4200 Porto, Portugal
}

\section{NOTATION}

d infinitesimal variation

$g$ molar Gibbs' free energy, $\mathrm{J} / \mathrm{mol}$

$G \quad$ Gibbs' free energy, J

$\hat{G}$ Gibbs' free energy for the integrated approach, J

$n \quad$ amount of matter, mol

$N \quad$ number of pairs of reactor and separator units

$\mathrm{P} \quad$ product

$\mathrm{R} \quad$ reactant

$R \quad$ ideal gas constant, $\mathrm{J} /(\mathrm{mol} . \mathrm{K})$

$T$ absolute temperature, $\mathrm{K}$

$x \quad$ molar fraction

$X$ molar conversion

\section{Greek letters}
$\Delta \quad$ finite variation
$\mu \quad$ chemical potential, $\mathrm{J} / \mathrm{mol}$
$\Xi \quad$ integration dummy variable

\section{Subscripts}

$0 \quad$ at inlet

$i \quad$ at the $i$ th reactor unit

eq at equilibrium

$P \quad$ product $P$

$P, T \quad$ isobaric and isothermal conditions

$r \quad$ chemical reaction

$\mathrm{R} \quad$ reactant $\mathrm{R}$

$r+s \quad$ single set of reactor/separator units

$R+S \quad$ multiple set of reactor/separator units

$S \quad$ physical separation

Superscript

$\theta \quad$ standard conditions

\section{REFERENCES}

Gradshteyn, I. S. and Ryzhik, I. M. (1980) Table on Integrals, Series, and Products. Academic Press, San Diego, U.S.A.

Laane, C., Tramper, J. and Lilly, M. D. (Eds) (1987) Biocatalysis in Organic Media. Elsevier, Amsterdam, The Netherlands.

Paiva, A. L., van Rossum, D. and Malcata, F. X. (1996) Kinetic improvement of chemical processing of an ideal binary mixture when enzyme-catalyzed reaction is integrated with physical separation. Biocatalysis Biosep. (sub mitted).

Smith, J. M. and van Ness, H. C. (1987) Introduction to Chemical Engineering Thermodynamics. McGraw-Hill, New York, U.S.A

Tramper, J., Vermue, M. H., Beeftink, H. H. and von Stockar, U. (Eds.) (1992) Biocatalysis in Non-conventional Media. Elsevier, Amsterdam, The Netherlands. 